\title{
TRANSIENT AND NON-TRANSIENT INTUSSUSCEPTIONS OF THE LARGE BOWEL IN ADULTS: TWO CASE REPORTS
}

\author{
René Vobořil ${ }^{1}$, Jan Fanta' ${ }^{1}$ Petr Bačkovský2, Daniel Ehrenberger ${ }^{2}$, Jana Vobořilováa
}

Department of Surgery, First Faculty of Medicine, Charles University in Prague and Na Bulovce Hospital, Prague, Czech Republic $^{1}$; Department of Surgery, Hospital of Merciful Sisters of St. Borromeo, Prague, Czech Republic ${ }^{2}$; Department of Biochemistry, Cell and Molecular Biology, Division of Cell and Molecular Biology, Third Faculty of Medicine, Charles University in Prague, Prague, Czech Republic ${ }^{3}$

Summary: Background: Intussusception of the large bowel in adults is a very rare pathological condition. However, it has its clinical importance because intussusception is very often associated with an intraluminal lesion. Case report: We report two cases of the large bowel intussusception, ileocolic and colorectal. Both intussusceptions were associated with a malignant tumor. However, the clinical presentation was different. One of the intussusceptions was of non-transient character, while the second one resolved spontaneously before operation. Both patients underwent surgery and malignant tumors were found and removed. Results: The purpose of the article is to draw attention to intussusception and emphasize that intussusception, either transient or non-transient, should be further examined. Conclusions: The intussusception may be the first and the only signal of the existence of a malignant tumor, very often colorectal carcinoma.

Keywords: Intussusception; Transient; Large bowel; Colorectal cancer; Surgery

\section{Introduction}

Intussusception, the invagination of the proximal segment of the gastrointestinal tract (intussusceptum) into an adjacent distal segment (intussuscipiens) is a pathological condition that may involve any part of the gastrointestinal tract. It is a diagnosis relatively often seen in children, while in adults not. Adult intussusception accounts for $5 \%$ of all intussusceptions and for $1 \%$ of all bowel obstructions (1).

Intussusception of adults most frequently occurs in the small intestine. Involvement of the large bowel is rare and often associated with an intraluminal lesion. Majority of patients has a benign or malignant tumor.

We report two cases of adult intussusception of the large bowel. Both of them are associated with advanced carcinoma with the primary site in the large bowel. Moreover, one of them represents a very unusual diagnosis of transient intussusception, which resolved spontaneously before operation.

\section{Case report 1}

A 69-year-old woman was admitted to department of gastroenterology for colic abdominal pain lasting approximately two weeks. Her personal clinical history was negative. At the admission, she denied history of weight loss, vomiting, and stoppage of intestinal passage.

On the clinical examination palpable sensitive resistance of the middle epigastrium was present, however there were no signs of peritoneal irritation. CT examination of the abdominal cavity (Fig. 1) revealed voluminous ileocolic intussusception $160 \times 160 \mathrm{~mm}$ in size with a small amount of free fluid in the Douglas' cul-de-sac.

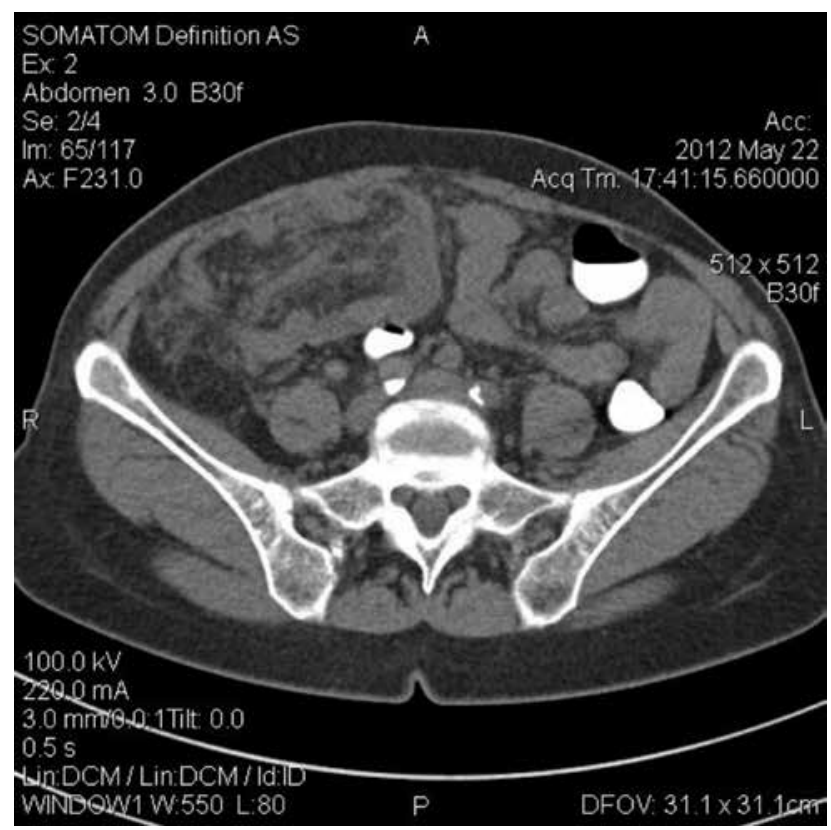

Fig. 1: Abdominal CT before operation in patient 1 showing voluminous ileocolic intussusception. 
At surgery, the abdominal cavity was open using middle laparotomy. As described on the CT scans, in the area of the right mesogastrium the ileocolic intussusception was visible. Inside of the intussusception tumor resistance was palpable. Probably, the lead point of the intussusception was the tumor, which together with the ileocecal area and appendix, was intussuscepted into the ascending colon. The tumor was $5.5 \times 4.5 \mathrm{~cm}$ in size.

There were no enlargement of the intestine loops and no signs of ileus as well as no signs of tumor dissemination in the abdominal cavity and in the liver. The right hemicolectomy with one layer side-to-side ileo-transverse anastomosis was performed.

The postoperative course was without any complications. In the eleventh postoperative day the patient was discharged home. Histopathological examination revealed malignized tubovillous adenoma with poorly differentiated adenocarcinoma, localized near to the ileocecal valve in the large bowel. Invasion depth was the layer of submucosis and muscularis propria. Two regional lymph nodes were positive for metastatic adenocarcinoma infiltration. The patient was sent to oncology department for subsequent anticancer treatment and follow-up.

\section{Case report 2}

A 60-year-old man without any co-morbidity underwent colonoscopy because of rectal bleeding with result of a rectal tumor in a distance of $5 \mathrm{~cm}$ from the anus. The tumor blocked the whole lumen of the rectum thus it was not possible to get through with the endoscope. The histological examination of the tissue sample obtained from the endoscopy revealed high-grade dysplasia with suspicion of cancer.

At surgery department the patient underwent physical examination. No subjective complaints were observed. There were no signs of stoppage of intestinal passage. Digital per rectum examination showed voluminous rectal tumor filling in the whole rectal ampulla. The tumor was tightly fixed to the surrounding tissues.

CT demonstrated (Fig. 2) a surprising finding, a voluminous colorectal intussusception $50 \times 60 \times 150 \mathrm{~mm}$ in size The sigmoid colon and mesocolon were invaginated into the rectum. On the CT scans any potential pathological mass localized inside of the invagination could not be identified. No signs of tumor dissemination were found.

The patient was planned for elective surgery with revision of the abdominal cavity. Immediately before operation, digital per rectum examination detected no tumor in the rectum. At surgery, any intussusception was not present. Instead of the situation described on the CT scans, the dolichosigma with a tumor $3 \times 3 \mathrm{~cm}$ in size situated on the top of the sigmoideal loop was found. A thickening of the large bowel wall distal from the tumor was visible. There were no signs of tumor dissemination in the abdominal cavity. The dolichosigma with the tumor was resected and two layer end-to-end anastomosis was performed. Because the large

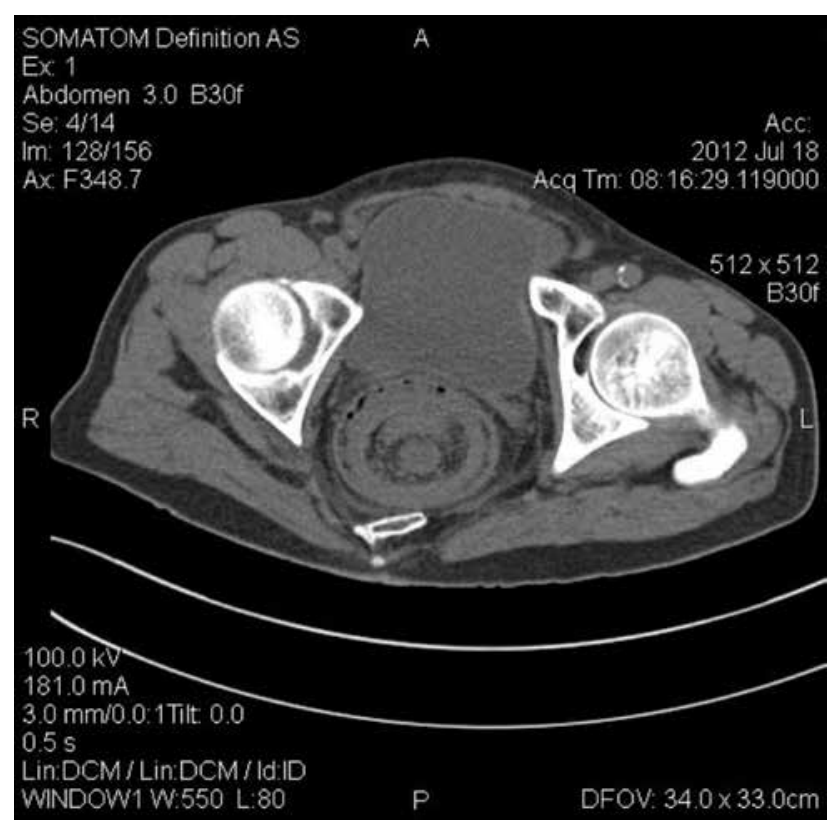

Fig. 2: Abdominal CT before operation in patient 2 showing voluminous colorectal intussusception. The sigmoid colon and mesocolon are invaginated into the rectum.

bowel was thickened, to be sure that the anastomosis will heal properly, the anastomosis was accompanied by a protective transversostomy.

The patient had a full recovery. In the eleventh postoperative day he was discharged home. Three months later, the patient was reoperated to close the protective transversostomy. The operation and postoperative course were without any complications.

The disease was histopathogically diagnosed as an advanced sigmoid colon cancer. It was mucinous adenocarcinoma with the diameter of $4.5 \mathrm{~cm}$ in size with surface ulceration. Invasion depth was pericolic adipose tissue. The regional lymph nodes were negative for cancer cells. The patient was recommended for further oncological examination and treatment.

\section{Discussion}

Adult intussusception is a very rare and challenging condition, especially when located in the large bowel. It requires preoperative diagnostic skills, careful and considerate intra-operative judgment, and appropriate postoperative follow-up in order to anticipate a successful outcome (2).

The exact mechanism of origin of intussusception is still unknown. It is supposed that any lesion in the bowel wall or irritant within the lumen of the bowel that alters normal peristaltic activity is able to initiate intussusception. Ingested food and subsequent peristaltic activity of the bowel produce an area of constriction above the stimulus and relaxation below, thus telescoping the lead point through the lumen of the distal bowel (2). 
Some intussusceptions may resolve spontaneously without surgical intervention. Such intussusceptions are called transient. There are only a few articles describing transient intussusception. One of them published by Gollub (3) presents comprehensive study on intussusception of the large bowel at a single cancer centre. From 34 intussusceptions, seven were transient caused by lipomas ( 2 patients), edema due to portal hypertension in patient with cholangioma, colon cancer, tubovillous adenoma, presumed sarcoma and presumed carcinoid. More often there are data published on transient intussusception of the small bowel. Most of them are seen in patients diagnosed with Crohn disease (4) and celiac disease (5). Transient intussusception has also been described as an incidental finding without any defined underlying cause for intussusception in patients with different basic diagnoses such as aortic aneurysm, hepatic cyst or cerebral metastases (6). It seems to be this phenomenon probably functional since none of the cases had defined cause for the intussusception $(6,7)$.

The clinical presentation of adult colonic intussusception is mostly subacute or chronic, lasting several days or weeks. Although acute presentations do occasionally occur as well. The symptomatology is often unspecific and may vary among individuals, therefore it is impossible to define an exact diagnostic algorithm. Most patients have nonspecific symptoms that are related to the gastrointestinal tract. Abdominal pain is the most common symptom, followed by vomiting and nausea (2). Less frequently melena, weight loss, fever, and constipation may occur. In our cases, the first patient presented with two weeks long colic abdominal pain and no other symptoms. The second patient suffered from rectal bleeding. He was suspected of having voluminous low rectal tumor and the intussusception was surprising finding on CT.

Several imaging techniques may help to make a diagnosis of intussusception. There are employed examination methods, such as plain abdominal X-rays, barium enema examination, colonoscopy, ultrasonography and CT. In last years, CT has been reported to be the most useful tool for diagnosis of intussusception and is superior to other examination methods (8). CT can reliably diagnose intussusception because of its virtually pathognomonic appearance. According Yakan et al. (8) the diagnostic accuracy of CT scans was $83.3 \%$ in their study. In our two cases, the diagnosis was made by $\mathrm{CT}$ examination.

Treatment of the large bowel intussusception usually requires surgery since we have to think about possibility of an underlying pathologic process with possible malignant ori- gin that may act as a lead point. This was seen in our patients, advanced colorectal carcinoma was a part of the telescoped bowel in both cases. Therefore we may suppose that both carcinomas served as the lead points of the intussusceptions. Such situation can be resolved only by a surgical intervention. However, there is not a clear consensus about the optimal surgical approach and there is still controversy about reduction before resection (9). Most authors prefer resection without previous reduction of intussusception, because reduction may augment the risk of bowel perforation or dissemination of malignant cells. However, according some authors (10) it is sometimes possible to reduce intussusception by simple manipulation or by compression.

In case that the intussusception is associated with a tumor and the final histological examination confirmed malignancy, the patient should be referred to department of oncology for consideration of further examination, anticancer treatment and follow-up.

In conclusion, diagnosis of the intussusception of the large bowel, either transient or non-transient, should be further examined. The intussusception may be the first and the only signal of the existence of a malignant tumor, very often colorectal carcinoma. Thus, in case of the transient intussusception without any other subjective problems of a patient, presence of potential pathologic mass in the large bowel should be excluded. The non-transient intussusception should be solved by surgery.

\section{References}

1. Eisen LK, Cunningham JD, Aufses AH. Intussusception in adults: institutional review. J Am Coll Surg 1999; 188: 390-395.

2. Begos DG, Sandor A, Modlin IM. The diagnosis and management of adult intussusception. Am J Surg 1997; 173: 88-94.

3. Gollub MJ. Colonic intussusception: clinical and radiographic features. AJR Am J Roentgenol 2011; 196: W580-585.

4. Knowles MC, Fishman EK, Kuhlman JE, Bayless TM. Transient intussusception in Crohn disease: CT evaluation. Radiology 1989; 170: 814

5. Willingham FF, Opekun AR, Graham DY. Endoscopic demonstration of transient small bowel intussusception in a patient with adult celiac disease. Gastrointest Endosc 2003; 57: 626-627.

6. Catalano O. Transient small bowel intussusception: $\mathrm{CT}$ findings in adults. $\mathrm{Br} \mathrm{J}$ Radiol 1997; 70: 805-808.

7. Zissin R, Gayer G, Konen O, Shapiro-Feinberg M. Transient colocolic intussusception. Clin Imaging 2000; 24: 8-9.

8. Yakan S, Caliskan C, Makay O, Denecli AG, Korkut MA. Intussusception in adults: clinical characteristics, diagnosis and operative strategies. World J Gastroenterol 2009; 15: 1985-1989.

9. Erkan N, Haciyanli M, Yildirim M, Sayhan H, Vardar E, Polat AF. Intussusception in adults: an unusual and challenging condition for surgeons. Int J Colorectal Dis 2005; 20: 452-456.

10. Yol S, Bostanci EB, Ozogul Y, Akoglu M. Extensive adult colo-colonic intussusception from ascending colon to sigmoid colon: report of a case. Turk J Gastroenterol 2004; 15: 201-203

Received: 19/04/2015

Accepted in revised form: 18/05/2015

\section{Corresponding author:}

Assoc. Prof. René Vobořil, MD, PhD., Department of Surgery, First Faculty of Medicine of the Charles University in Prague and Na Bulovce Hospital, Budinova 67/2, 18081 Prague, Czech Republic; e-mail: rene.voboril@seznam.cz 